\title{
Comparison of Antibacterial Efficacy of Cinnamon Extract and Calcium Hydroxide as Intracanal Medicament against E. fecalis: An in vitro Study
}

\author{
Veerale Panchal', Deepa Gurunathann ${ }^{1,}$, Lakshmi Thangavelu²
}

\section{Veerale Panchal ${ }^{1}$, Deepa Gurunathan ${ }^{1, *}$, Lakshmi Thangavelu}

'Department of Pediatric and Preventive Dentistry, Saveetha Dental College, Saveetha Institute of Medical and Technical Sciences, Chennai, Tamil Nadu, INDIA.

${ }^{2}$ Department of Pharmacology, Saveetha Dental College, Saveetha Institute of Medical and Technical Sciences, Chennai, Tamil Nadu, INDIA.

Correspondence

Deepa Gurunathan

Professor, Department of Pediatric and Preventive Dentistry, Saveetha Dental College, Saveetha Institute of Medical and Technical Sciences, Chennai, Tamil Nadu, INDIA.

Phone no : +919994619386

E-mail: drgdeepa@yahoo.co.in

History

- Submission Date: 13-05-2018.

- Review completed: 04-06-2018;

- Accepted Date: 11-07-2018

DOI : 10.5530/pj.2018.6.199

Article Available online

http://www.phcogj.com/v10/i6

\section{Copyright}

(c) 2018 Phcog.Net. This is an openaccess article distributed under the terms of the Creative Commons Attribution 4.0 International license.

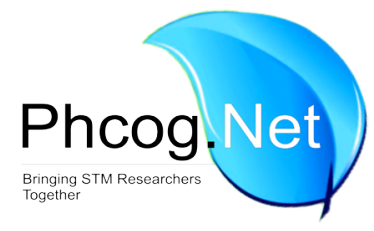

\begin{abstract}
Background: There has been an increased use of natural products as medicament in endodontic treatment due to the increased adverse effect observed in chemical products used. The natural products used aims to disinfect the root canal and provide a sterile environment as efficiently as the comparable chemical products. Aim: To compare the antibacterial efficacy of cinnamon extract and calcium hydroxide as intracanal medicament against $E$. fecalis. Materials and Methods: The study is an in vitro randomized controlled study conducted on 40 single rooted permanent teeth. The study sample was divided into two groups- cinnamon extract as intracanal medicament and calcium hydroxide. The intracanal medicament was placed for 7 days. The outcome was measures using colony forming units per $\mathrm{ml}$. Statistical analysis was done using Mann-Whitney's test to compare between the two groups. Results: Cinnamon extract intracanal medicament showed higher antibacterial efficacy as compared to calcium hydroxide. $(p=0.002)$. Conclusion: Cinnamon extract showed better antimicrobial efficacy against $E$. feacalis as intracanal medicament as compared to calcium hydroxide.

Key words: Cinnamon extract, Calcium hydroxide, Intracanal medicament, E. feacalis, Antibacterial efficacy.
\end{abstract}

\section{INTRODUCTION}

Bacterial colonization in the root canal systems of the teeth is a major source of infection causing tooth pain and periapical involvement. ${ }^{1}$ Wide number of bacteria have been isolated from the root canal systems. ${ }^{2}$ The root canal microflora of the infected teeth consists of gram positive, gram negative and anaerobic bacteria. ${ }^{2}$ Out of all the bacterial species, Enterococcus faecalis (E. fecalis) is the most commonly associated bacteria species with periapical infection and re-infection of treated root canal. ${ }^{3}$

E. faecalis is a dominant enterococcus species, responsible for $80-90 \%$ of human enterococcus infection. ${ }^{4}$ The virulence factor of $E$. faecalis is associated with its ability to penetrate the dentinal tubules and adhere to the collagen in the presence of human serum. ${ }^{5}$ Another important factor is the ability of the survival of this bacteria even under low nutrition environment. ${ }^{5}$ E. faecalis also shows resistance to various intracanal medicaments ${ }^{6}$ and has an ability to survive in the absence of any other microbes. ${ }^{7}$ Thus, elimination of E. faecalis is of utmost importance in complete disinfection and success of root canal treatment.

Biomechanical preparation and irrigation of root canals are the primary steps for root canal disinfection. ${ }^{8}$
However, due to anatomic complexity of permanent root canal system, complete disinfection by biomechanical preparation alone is not possible. Hence, addition of intracanal medicaments acts as an additional aid for the complete disinfection of root canal system. ${ }^{6}$ The additional antibacterial effectiveness of intracanal medicament is attributed to its disinfection by penetration in the dentinal tubules. ${ }^{9}$

Calcium hydroxide is the most common intracanal medicament used. The antibacterial activity of calcium hydroxide is due to its high alkalinity. ${ }^{10}$ The antibacterial activity is related to the release of hydroxyl ions. ${ }^{10}$ The hydroxyl ions show antibacterial activity by lysis of the bacterial cell wall, damage to bacterial cytoplasm, protein denaturation and damage to the DNA in bacteria. However, studies have shown limited antimicrobial spectrum of calcium hydroxide. ${ }^{10-11}$ Also, cases of root fracture due to long term use of calcium hydroxide have been reported. ${ }^{12}$ To overcome the disadvantage of calcium hydroxide and prevent antimicrobial resistance, various natural alternatives have been tested recently for their antimicrobial action. ${ }^{13-15}$ Vasudeva et al. showed substantial antibacterial effectiveness of honey, 
Panchal, et al.: Cinnamon extract medicament against E. fecalis

aloe Vera gel, propolis gel and curcuma longa gel as intracanal medicaments against E. faecalis. ${ }^{16}$ Madhubala et al. showed effectiveness of propolis as an intracanal medicament against E. faecalis. ${ }^{17}$ These natural products have shown promising results as antibacterial agents.

Cinnamon also known as C. zeylanicum belongs to the Lauraceae family. ${ }^{18}$ Extracts for the same is obtained from the leaf, bark, fruit and flowers. ${ }^{19}$ The antibacterial activity exhibited by cinnamon is due to the presence of vanillic, caffeic, gallic, protocatechuic, p-coumaric, and ferulic acids in the extracts. ${ }^{19}$

Studies have evaluated the antibacterial activity of cinnamon against $E$. faecalis..$^{20-21}$ To the knowledge of the authors the antibacterial efficacy of cinnamon extract as an intracanal medicament has not been evaluated. In this regard, the present study aims to compare the antimicrobial property of the cinnamon extract as an intracanal medicament with calcium hydroxide by determining the colony forming units of $E$. faecalis in extracted teeth.

\section{MATERIALS AND METHODS}

The present study followed a randomized control in vitro study design. The study protocol was approved by the institutional review board of Saveetha dental college. The present study was a single blinded study. Two different personnel were assigned for the study. The shaping of the canals and placement of intracanal medicament was done by one single operator. The antibacterial efficacy was evaluated using colony forming units. The colonies were counted by a separate operator who was unaware of the two study groups.

The present study uses ethanolic extract of cinnamon to prepare the intracanal medicament. ${ }^{22}$ The ethanolic extract obtained from cinnamon was obtained from cinnamon bark grounded into coarse and medium coarse particles. These particles were mixed with $50 \%$ ethanol and the suspension was placed on the magnetic stirrer for $4 \mathrm{~h}$. The suspension was filtered using Whitman's filter paper and then dried to obtain the extract. This prevents the influence of extracting agent (ethanol) on the final extract. After preparation, the cinnamon extract was stored extracts in dark colored bottles at 4 degrees Celsius until use.

Intracanal medicament was prepared by mixing crude cinnamon extract with propylene glycol. According to a previous study cinnamon showed Minimum inhibitory concentration at $20 \%{ }^{21}$ Hence a $20 \%$ cinnamon was prepared by mixing $2 \mathrm{~g}$ of cinnamon in $10 \mathrm{ml}$ of propylene glycol. This was a freshly prepared mixture prepared immediately before use. Another group of the study used calcium hydroxide injectable paste (Calcicur, VOCO, Cuxhaven)

The present study includes a total of 40 extracted single rooted permanent teeth which were extracted for orthodontic purpose or due to any periodontal problems. Teeth with dental caries, dental caries with pulpal involvement, root fracture, curved canals or anatomic malformation were not included in the study. Any tissue tags, calculus or debris present on the external tooth surface was removed to obtain a clean surface. These teeth were stored in normal saline until use to prevent desiccation.

The extracted teeth were mounted on individual block of modelling wax. Access was opened using with No. 4 round carbide bur (DENTSPLY Maillefer, OK, USA). The dentinal overhangs of the roof of the pulp chamber was removed used a safe ended tungsten carbide bur (Endo-Z, FG, DENTSPLY Maillefer, OK, USA). The patency of the canals was checked using No 20 stainless steel K- file (DENTSPLY Maillefer, OK, USA). The working length was determined using a pre-operative radiograph and was kept $0.5 \mathrm{~mm}$ short of the radiographic apex. Initial instrumentation was done up to no 30 stainless steel hand files which was followed by rotary instrumentation using ProTaper (DENTSPLY
Maillefer, OK, USA) up to F3. ProTaper files were used in their sequential order. Saline was used in between the instrumentation sequence to remove the debris. After complete instrumentation the tooth were scheduled for the inoculation of E. faecalis.

Prior to the inoculation of E. faecalis in the teeth, they were autoclaved twice at 121 degree celcius for the elimination of microbes from the teeth and prevent contamination of the study with other microbes. A pure culture of test strain of E. faecalis ATCC 29212 (Department of Microbiology, PGIMS) was inoculated in sterile nutrient broth. The presence of $E$. faecalis was confirmed in the nutrient broth by pipetting 10 micro liter of the broth and observing its presence under microscope. Nutrient broth inoculated with $E$. faecalis was transferred in a sterile container where the level of the broth was marked. The extracted teeth placed in the nutrient broth containing E. faecalis and incubated for 21 days at 37 degree Celsius. The level of the nutrient broth was checked daily, and new broth inoculated with $E$. faecalis was added to maintain all the extracted teeth completely submerged in the inoculated broth for 21 days.

The inoculated teeth were divided into two groups for the irrigation protocol. 20 teeth were randomly assigned to each group. Each tooth was assigned a serial number. The allocation of each sample into individual groups was done using block randomization using lots. Cinnamon extract intracanal medicament paste was placed using rotary lentulospiral in Group 1. Calcium hydroxide paste was injected in the canal in Group 2. The canal was uniformly filled with calcium hydroxide using rotary lentulospiral. The intracanal medicament was left in place and the tooth was sealed using temporary cement (Cavity-G, 3M ESPE, United States) for 7 days. The tooth was wrapped in moist cotton and kept in incubator at 37 degree Celsius.

After 7 days the temporary filling was removed, and the sample of dentin was taken to evaluate the reduced colony forming units. No 2 GatesGlidden drill was used to remove the dentin sample from each tooth. A fresh sterilized Gates Glidden drill was used for every tooth to prevent cross-contamination. The dentin debris from the Gates Glidden drill was transferred to $1 \mathrm{ml}$ of saline. All the micro-test tubes were kept in water bath for $30 \mathrm{~min}$ at 62 degree Celsius to prevent cross- contamination. 25 microliters of the solution from the micro test tubes were pipetted and inoculated on the nutrient agar. This agar was incubated at 37 degree Celsius and the colonies of E. faecalis was calculated under magnifying glass. The presence of E. faecalis was verified using eosinophil stain and gentian violet staining to identify gram positive microorganism.

The data obtained were entered in a excel sheet and was analyzed using SPSS software version 22. (IBM Corp, Armont, NY, USA) Mann-Whitney's test was used for comparison between the two groups.

\section{RESULTS}

A total of 40 permanent single rooted teeth are included in the study. Out of the total teeth included 31were single rooted lower premolars. 5 were upper central incisors and 4 were lateral incisors. Out of the 20 teeth filled with cinnamon extract as intracanal medicament, 17 were premolars, 2 central incisor and 1 lateral incisor. Out of the 20 teeth filled with calcium hydroxide, 14 were premolars, 3 central incisors and 3 lateral incisors. The outcome variable for all the groups is the CFU values per $\mathrm{ml}$.

The Normality tests Kolmogorov-Smirnov and Shapiro-Wilks tests results reveal that the variable follows a non-normal distribution of data. Therefore, to analyze the data non-parametric methods are applied.

The mean CFU values obtained using cinnamon as intracanal medicament was $0.21 \times 10^{3}$ having a standard deviation of 0.14 . The mean CFU values obtained using calcium hydroxide as intracanal medicament was $0.77 \times 10^{3}$ having a standard deviation of 0.40 . 
Table 1: Comparison of CFU values per $\mathrm{ml}$ between the cinnamon intracanal medicament and calcium hydroxide group.

\begin{tabular}{cccccc}
\hline Sr.no & Groups & $\begin{array}{c}\text { Number } \\
\text { of sample }\end{array}$ & Mean & $\begin{array}{c}\text { Standard } \\
\text { Deviation }\end{array}$ & P-value \\
\hline 1 & $\begin{array}{c}\text { Cinnamon } \\
\text { extract }\end{array}$ & 20 & $0.21 \times 10^{3}$ & 0.146 & $0.002^{*}$ \\
2 & $\begin{array}{c}\text { Calcium } \\
\text { hydroxide }\end{array}$ & 20 & $0.77 \times 10^{3}$ & 0.401 & \\
\hline
\end{tabular}

*significant- $P<0.05$. Mann- Whitney's test.

\section{Cinnamon medicament}

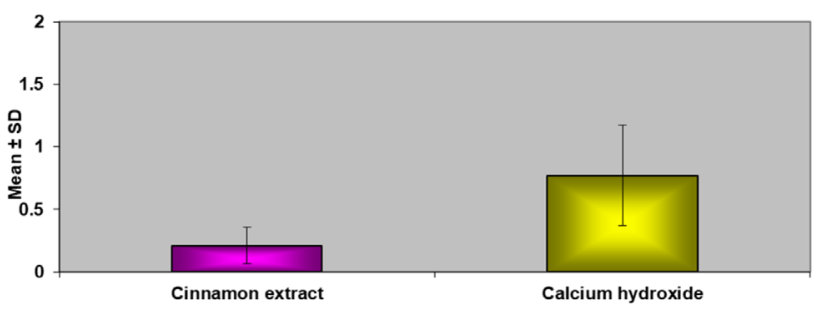

Figure 1: Comparison of CFU values per ml between the cinnamon intracanal medicament and calcium hydroxide group.

Mann-Whitney's test was used to compare CFU values between the two groups. Mann-Whitney's test showed a significant difference between the two groups with cinnamon having less CFU values as compared to calcium hydroxide $(p=0.002)$. (Table 1, Figure 1)

Thus, intracanal medicament using cinnamon extract formed less colonies of $E$. faecalis as compared to calcium hydroxide intracanal medicament.

\section{DISCUSSION}

Elimination of microbes and complete disinfection of the root canal is the ultimate goal of a successful endodontic treatment. ${ }^{3}$ Biomechanical preparation alone cannot eliminate all the microbes from the root canal. In clinical cases of infected root canal and periapical infection, an addition of medicament would aid in increased penetration into the dentin thus providing an extended antibacterial effect. ${ }^{23}$

Calcium hydroxide is the most commonly used intracanal medicament in teeth with periapical lesion. ${ }^{24}$ However, use of calcium hydroxide pose an additional risk of internal tooth resorption and root fracture..$^{25}$ Antibiotics also have gained popularity as intracanal medicament and have shown higher antibacterial effect than calcium hydroxide. ${ }^{26}$ However, use of antibiotics subjects the patient at the risk of developing resistance. ${ }^{27}$ Hence, the use of herbal alternatives having similar antibacterial action will prove to be more beneficial. The present study uses cinnamon extract as an intracanal medicament and compares its antibacterial efficacy with the most commonly used medicament calcium hydroxide.

Cinnamon extract has recently gained popularity in endodontics due to its antibacterial properties. E. faecalis being the most common cause of infection and reinfection in root canal, studies are conducted to test antibacterial effectiveness of cinnamon extract against $E$. faecalis. ${ }^{22,28}$ All these studies have used cinnamon extract as endodontic irrigant. None of the studies compare cinnamon extract as intracanal medicament.
Secondly, there is an increase use of another herbal agent as intracanal medicament. Kurian et al. showed mushroom and aloe Vera to have better antimicrobial properties as compared to calcium hydroxide medicament. ${ }^{29}$ Mustafa $\mathrm{M}$ showed comparable results between neem extract as intracanal medicament, sodium hypochlorite and chlorhexidine. ${ }^{30}$ Thus, the present study uses the principles of the previous studies to evaluate the antimicrobial efficacy of cinnamon as intracanal medicament.

Since no previous studies compares the antimicrobial effect of cinnamon as intracanal medicament, the present study follows an in vitro design. Randomization was followed to maintain a standard protocol for the study. However, due to the difference in the consistency of the medicament, only single personnel blinding was followed.

A concentration of $20 \%$ of the cinnamon extract was used as intracanal medicament. This concentration was based on the previous studies which showed Minimal inhibitory concentration for E. faecalis to be 1-20\%. ${ }^{31}$ The intracanal medicament was prepared by mixing $20 \%$ of cinnamon extract with propylene glycol. This was based on the study by Kurian et al. and Mustafa $\mathrm{M}$ who showed similar preparation methods for other herbal agents in their respective study. ${ }^{29-30}$ Propylene glycol was used as a medium as compared to distilled water because propylene glycol has showed increased penetration into the dentin as compared to distilled water. ${ }^{31}$ Rotary lentulospiral was used to deliver intracanal medicament into the canals. Previous study has shown uniform delivery of the intracanal medicament using lentulospiral. ${ }^{32}$

The intracanal medicament was placed in the canal for 7 days. Previous studies have shown antibacterial effectiveness of intracanal medicament in the duration of 7 days. ${ }^{33}$ Hence to have a similar protocol for both the study group, a similar duration of intracanal medicament was followed in the study.

The present study measures Colony forming units as outcome variable. Colony counts of isolated bacteria will provide a judgement of antibacterial effectiveness of an agent against another. However, in recent times more standardized methods like PCR and use of bifocal microscopy will give more reliable results.

According to the present study, cinnamon extract showing more inhibition to the colonies of E. feacalis as compared to calcium hydroxide. Calcium hydroxide is known to have a limited activity against $E$. feacalis. ${ }^{24}$ This can be one of the reasons for cinnamon extract to show better results. Previous studies of Kurian et al. ${ }^{29}$ showed herbal agents (aloe Vera and mushroom) to have better efficacy than calcium hydroxide. This increased antibacterial action can also be attributed to presence of antioxidants, vanillic, caffeic, gallic, protocatechuic, p-coumaric, and ferulic acids in the extract. Further studies evaluating the mechanism of action of the constituents of the herbal medicaments will aid in more detailed evaluation of the extract.

The present study thus shows better antibacterial effectiveness of cinnamon extract as compared to calcium hydroxide agents. This is however a preliminary study to evaluate the effectiveness of the new medicament. Further studies are required evaluating the effectiveness of the new medicament at the molecular level and understand the exact mechanism against E. fecalis.

\section{CONCLUSION}

The present study shows the antibacterial effectiveness of cinnamon extract as intracanal medicament in permanent teeth infected with E. feacalis. The present study shows better antibacterial effectiveness of cinnamon extract as intracanal medicament as compared to calcium hydroxide. Thus, cinnamon extract can be used as an herbal alternative to other chemical agents used for disinfection of the root canal. 


\section{ACKNOWLEDGEMENT}

Acknowledging Department of Microbiology, Saveetha Dental College for providing the bacterial strain and equipment for culture. Acknowledging Green Chem, Bengaluru for providing cinnamon extract.

\section{CONFLICT OF INTEREST}

None

\section{ABBREVIATIONS}

ATCC: American type culture collection; C. zeylanicum: Cinnamomum zeylanicum; DNA: Deoxyribonucleic acid; E. faecalis: Enteroccocus faecalis; g: Grams.

\section{REFERENCES}

1. Kakehashi S, Stanley HR, Fitzgerald RJ. The Effects Of Surgical Exposures Of Dental Pulps In Germ-Free And Conventional Laboratory Rats. Oral Surg Oral Med Oral Pathol. 1965;20(1):340-9.

2. Gomes BPFA, Pinheiro ET, Gadê-Neto CR, Sousa ELR, Ferraz CCR, Zaia AA, et al. Microbiological examination of infected dental root canals. Oral Microbiol Immunol. 2004;19(2):71-6.

3. Gomes BPFA, Pinheiro ET, Sousa ELR, Jacinto RC, Zaia AA, Ferraz CCR, et al. Enterococcus faecalis in dental root canals detected by culture and by polymerase chain reaction analysis. Oral Surg Oral Med Oral Pathol Oral Radio Endod. 2006;102(2):247-53.

4. Ruoff KL, Maza LDL, Murtagh MJ, Spargo JD, Ferraro MJ. Species identities of enterococci isolated from clinical specimens. J Clin Microbiol. 1990;28(3):435-7.

5. Love RM. Enterococcus faecalis-a mechanism for its role in endodontic failure. Int Endod J. 2001:34(5):399-405.

6. Byström A, Sundqvist G. Bacteriologic evaluation of the effect of 0.5 percent sodium hypochlorite in endodontic therapy. Oral Surg Oral Med Oral Pathol. 1983;55(3):307-12

7. Fabricius L, Dahlén G, Holm SE, Möller AJ. Influence of combinations of ora bacteria on periapical tissues of monkeys. Scand J Dent Res. 1982;90(3):200-6.

8. Love RM, Jenkinson HF. Invasion of dentinal tubules by oral bacteria. Crit Rev Oral Biol Med Off Publ Am Assoc Oral Biol. 2002;13(2):171-83.

9. Haapasalo M, Orstavik D. In vitro infection and disinfection of dentinal tubules. J Dent Res. 1987;66(8):1375-9.

10. Siqueira JF, Lopes HP. Mechanisms of antimicrobial activity of calcium hydroxide: a critical review. Int Endod J. 1999;32(5):361-9.

11. Estrela C, Pimenta FC, Ito IY, Bammann LL. In vitro determination of direct antimicrobial effect of calcium hydroxide. J Endod. 1998;24(1):15-7.

12. Kahler SL, Shetty S, Andreasen FM, Kahler B. The Effect of Long-term Dressing With Calcium Hydroxide on the Fracture Susceptibility of Teeth. J Endod. 2018 44(3):464-9

13. Behera S, Khetrapal P, Punia SK, Agrawal D, Khandelwal M, Lohar J. Evaluation of Antibacterial Activity of Three Selected Fruit Juices on Clinical Endodontic Bacterial Strains. J Pharm Bioallied Sci. 2017;9(Suppl 1):S217-21.

14. Borzini L, Condò R, Dominicis PD, Casaglia A, Cerroni L. Root Canal Irrigation: Chemical Agents and Plant Extracts Against Enterococcus faecalis. Open Dent J. 2016;10(1):692-703
15. Sinha DJ, Nandha KDS, Jaiswal N, Vasudeva A, Prabha TS, Pratap SU. Antibacterial Effect of Azadirachta indica (Neem) or Curcuma longa (Turmeric) against Enterococcus faecalis Compared with That of 5\% Sodium Hypochlorite or 2\% Chlorhexidine in vitro. Bull Tokyo Dent Coll. 2017;58(2):103-9.

16. Vasudeva A, Sinha DJ, Tyagi SP, Singh NN, Garg P, Upadhyay D. Disinfection of dentinal tubules with $2 \%$ Chlorhexidine gel, Calcium hydroxide and herbal intracanal medicaments against Enterococcus faecalis: An in-vitro study. Singapore Dent J. 2017;38(1):39-44.

17. Madhubala MM, Srinivasan N, Ahamed S. Comparative evaluation of propolis and triantibiotic mixture as an intracanal medicament against Enterococcus faecalis. J Endod. 2011;37(9):1287-9.

18. WHO Monographs on Selected Medicinal Plants - Volume 1 [Internet]. [cited 2018 Jan 7]. Available from: http://apps.who.int/medicinedocs/en/d/Js2200e/

19. Nabavi SF, Lorenzo AD, Izadi M, Sobarzo-Sánchez E, Daglia M, Nabavi SM. Antibacterial Effects of Cinnamon: From Farm to Food, Cosmetic and Pharmaceutical Industries. Nutrients. 2015;7(9):7729-48.

20. Gupta A, Duhan J, Tewari S, Sangwan P, Yadav A, Singh G, et al. Comparative evaluation of antimicrobial efficacy of Syzygium aromaticum, Ocimum sanctum and Cinnamomum zeylanicum plant extracts against Enterococcus faecalis: a preliminary study. Int Endod J. 2013;46(8):775-83.

21. Al-Mariri A, Safi M. In vitro Antibacterial Activity of Several Plant Extracts and Oils against Some Gram-Negative Bacteria. Iran J Med Sci. 2014;39(1):36-43

22. Nabavi SF, Lorenzo AD, Izadi M, Sobarzo-Sánchez E, Daglia M, Nabavi SM. Antibacterial Effects of Cinnamon: From Farm to Food, Cosmetic and Pharmaceutical Industries. Nutrients. 2015;7(9):7729-48.

23. Young GR, Parashos $P$, Messer $\mathrm{HH}$. The principles of techniques for cleaning root canals. Aust Dent J. 2007;52(1 Suppl):S52-63.

24. Kim D, Kim E. Antimicrobial effect of calcium hydroxide as an intracanal medicament in root canal treatment: a literature review - Part I. In vitro studies. Restor Dent Endod. 2014;39(4):241-52

25. Lee Y. Effect of calcium hydroxide application time on dentin. Restor Dent Endod. 2013;38(3):186.

26. Pai S, Pai AV, Thomas MS, Bhat V. Effect of calcium hydroxide and triple antibiotic paste as intracanal medicaments on the incidence of inter-appointment flare-up in diabetic patients: An in vivo study. J Conserv Dent. 2014;17(3):208.

27. Valverde ME, Baca P, Ceballos L, Fuentes MV, Ruiz-Linares M, Ferrer-Luque CM. Antibacterial efficacy of several intracanal medicaments for endodontic therapy. Dent Mater J. 2017:36(3):319-24.

28. Mathew J, Pathrose S, Kottoor J, Karaththodiyil R, Alani M, Mathew J. Evaluation of an Indigenously Prepared Herbal Extract (EndoPam) as an Antimicrobia Endodontic Irrigant: An Ex vivo Study. J Int Oral Health. 2015;7(6):88-91.

29. Kurian. Efficacy of calcium hydroxide, mushroom, and Aloe vera as an intracanal medicament against Enterococcus faecalis: An in vitro study [Internet]. [cited 2018 Jan 10]. Available from: http://www.endodontologyonweb.org/article. asp? issn=0970-7212; year $=2016$; volume $=28 ;$ issue $=2 ;$ spage $=137$; epage $=142 ;$ a ulast=Kurian

30. Mustafa M. Antibacterial Efficacy of Neem (Azadirachta indica) Extract against Enterococcus faecalis: An in vitro Study. J Contemp Dent Pract. 2016:17(10):791-4.

31. Cruz EV, Kota K, Huque J, Iwaku M, Hoshino E. Penetration of propylene glycol into dentine. Int Endod J. 2002;35(4):330-6.

32. Oztan MD, Akman A, Dalat D. Intracanal placement of calcium hydroxide: a comparison of two different mixtures and carriers. Oral Surg Oral Med Ora Pathol Oral Radiol Endod. 2002;94(1):93-7.

33. Cook J, Nandakumar R, Fouad AF. Molecular- and culture-based comparison of the effects of antimicrobial agents on bacterial survival in infected dentinal tubules. J Endod. 2007;33(6):690-2
GRAPHICALABSTRACT

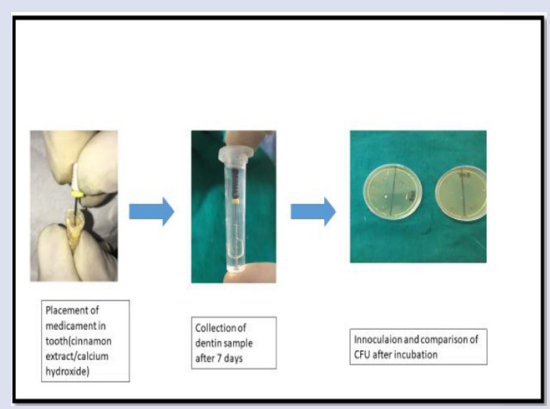

\section{SUMMARY}

- Calcium hydroxide is the commonly used intracanal medicament for the disinfection of root canals. However, due to its disadvantages, one of it being internal root resorption various alternatives have been suggested to effectively disinfect root canals. E. faecalis is the most common microorganisms present in root canals which leads to infection and reinfection of root canals.

- The present study is an in vitro study which compares effectiveness of calcium hydroxide and cinnamon extract as intracanal medicament against $E$. feacalis.

- The present study shows ethanolic cinnamon extract (20\% concentration) have statistically significant better antibacterial effectiveness as compared to calcium hydroxide when used as an intracanal medicament for a period of 7 days

Cite this article: Panchal V, Gurunathan D, Thangavelu L. Comparison of Antibacterial Efficacy of Cinnamon Extract and Calcium Hydroxide as Intracanal Medicament against E. fecalis: An in vitro Study. Pharmacog J. 2018;10(6):1165-8. 